\title{
Vitrification procedure decreases inositol 1,4,5-triphosphate receptor expression, resulting in low fertility of pig oocytes
}

\author{
J. Ito ${ }^{1,2}$, M. Hirose ${ }^{1}$, A. Nakamura ${ }^{1}$, M. Kamoshita ${ }^{1}$, T. Kato ${ }^{1}$, R. \\ Wojcikiewicz ${ }^{3}$, J.B. Parys ${ }^{4}$, N. Kashiwazaki ${ }^{1,2}$ \\ ${ }^{1}$ Graduate School of Veterinary Sciences, ${ }^{2}$ School of Veterinary Medicine, Azabu University, \\ Sagamihara, Kanagawa, 252-5201, Japan; ${ }^{3}$ Department of Pharmacology, State University of New \\ York, Upstate Medical University, Syracuse, NY 13120, USA; ${ }^{4}$ Laboratory of Molecular and Cellular \\ Signaling, Department of Cellular and Molecular Medicine, KU Leuven, Campus Gasthuisberg, O\&N1 \\ bus 00802, B-3000 Leuven, Belgium
}

Although cryopreservation of mammalian oocytes is an important technology, it is well known that unfertilized oocytes, especially in pigs, are highly sensitive to low temperature and that cryopreserved oocytes show low fertility and developmental ability (Wu et al. 2006). To avoid these detrimental effects caused by exposure to low temperature, many attempts have been done. It is recognized that one of the important issues contributing to successful vitrification is to maximize the rate of cooling and warming (Kuwayama et al. 2007). In our previous study, we demonstrated that Cryotop yields high post-warming survival and developmental ability of vitrified oocytes in the mouse (Kohaya et al. 2011) and rat (Fujiwara et al. 2010).

As for vitrification of metaphase-II (MII) oocytes, it is thought that kinetics of intracellular calcium is also an essential factor for the success. During fertilization, rises of intracellular calcium released from the endoplasmic reticulum via inositol 1,4,5-triphosphate receptor, $\left(\mathrm{IP}_{3} \mathrm{R} 1\right)$ occur in oocytes (Ito et al. 2010; Ito \& Kashiwazaki 2012). The rises of intracellular calcium induce exocytosis of cortical granules, resulting in the occurrence of zona hardening known as the zona reaction (Ducibella et al. 1988). In the mouse, Larman et al. (2006) demonstrated that exposure to vitrification solution containing cryoprotective agents causes the rise of intracellular calcium in MII oocytes, inducing zona hardening as well as normal fertilization. Indeed, our previous data showed that vitrification using calcium-free media significantly improved penetration and fertilization rates of vitrified oocytes in the mouse (Kohaya et al. 2011) and rat (Fujiwara et al. 2010). However, in pigs, the effect of calcium during vitrification in not well examined. In the present study, we focused on the kinetics of $I P_{3} R 1$ during vitrification in pig oocytes and tried to clarify the reason why vitrified pig oocytes show low fertility and developmental ability.

Cumulus oocyte complexes (COCs) were cultured for $44 \mathrm{~h}$ and then used for vitrification. Vitrification-warming was carried out by the Cryotop method as previously described (Fujiwara et al. 2010). Vitrified-warmed COCs were used for in vitro fertilization (IVF). For immunostaining, the germinal vesicle (GV) stage and MII stage oocytes were collected at $0 \mathrm{~h}$ and $44 \mathrm{~h}$, respectively. For western blotting, GV, MII, exposed MII and vitrified-warmed MII oocytes were collected. In vitro matured COCs were vitrified with Cryotop and then evaluated for fertility through IVF. Pronuclear formation rates of vitrified-warmed oocytes were significantly $(\mathrm{p}<$ 0.05) lower regardless of calcium supplementation $(9.0 \pm 1.3 \%(\mathrm{Ca}+)$ and $8.0 \pm 2.6 \%(\mathrm{Ca}-))$ compared to that of fresh ones $(62.4 \pm 13.0 \%)$. Most of the fertilized fresh oocytes reached the 2-cell stage $(53.3 \pm 10.1 \%)$. Some of the 2-cell embryos also developed to blastocysts (22.4 \pm $1.8 \%)$. However, none of the fertilized oocytes in the vitrified groups developed to the 2-cell 
stage. The penetrated sperm was confirmed in most of the oocytes as failing to form pronuclei, suggesting the possibility that vitrified-warmed oocytes have a decreased activity to induce calcium oscillations. To clarify the reason why vitrified-warmed oocytes had low fertility after IVF, localization of IP $R 1$ in vitrified-warmed oocytes was examined. In fresh MII oocytes, IP ${ }_{3}$ 1 is widely localized in oocyte cytoplasm and at the plasma membrane. Exposure to equilibration and vitrification media did not affect the IP ${ }_{3}$ R1 localization. However, in vitrified oocytes, no localization of $I P_{3} R 1$ at the plasma membrane could be visualized. These results suggest that the vitrification procedure may affect the expression of $I P_{3} R 1$ in pig oocyte. Therefore next, we tried to clarify whether the vitrification procedure affected $I P_{3} R 1$ expression in pig oocytes at the protein levels using western blotting. There were no significant differences in expression levels of IP $R 1$ and $\beta$-actin between GV and MII oocytes. In addition, exposure of oocytes to equilibration and vitrification solution did not affect expression levels of IP $R 1$ and $\beta$-actin. However, in vitrified-warmed oocytes, a significant decrease of $I P_{3} R 1$ expression was observed, while expression of $\beta$-actin was only slightly decreased.

Taken together, our present results demonstrate a possibility that the low fertility and developmental ability of vitrified MII porcine oocytes are due to a decreased expression of $I P_{3} R 1$ and its abnormal localization.

\section{References}

Ducibella T, Anderson E, Albertini DF, Aalberg J \& Rangarajan S 1988. Quantitative studies of changes in cortical granule number and distribution in the mouse oocyte during meiotic maturation. Developmental Biology 130 184-197.

Fujiwara K, Sano D, Seita Y, Inomata T, Ito J \& Kashiwazaki N 2010. Ethylene glycol-supplemented calcium-free media improve zona penetration of vitrified rat oocytes by sperm cells. Journal of Reproduction and Development 56 169-175.

Ito J \& Kashiwazaki N 2012. Molecular mechanism of fertilization in the pig. Animal Science Journal 83 669682.

Ito J, Parrington J \& Fissore RA 2011. PLCzeta and its role as a trigger of development in vertebrates. Molecular Reproduction and Development 78 846-853.
Kohaya N, Fujiwara K, Ito J \& Kashiwazaki N 2011. High developmental rates of mouse oocytes cryopreserved by an optimized vitrification protocol: the effects of cryoprotectants, calcium and cumulus cells. Journal of Reproduction and Development 57 675-680.

Kuwayama M 2007. Highly efficient vitrification for cryopreservation of human oocytes and embryos: the Cryotop method. Theriogenology 67 73-80.

Larman MG, Sheehan CB \& Gardner DK 2006. Calciumfree vitrification reduces cryoprotectant-induced zona pellucida hardening and increases fertilization rates in mouse oocytes. Reproduction 131 53-61.

Wu C, Rui R, Dai J, Zhang C, Ju S, Xie B, Lu X \& Zheng X 2006. Effects of cryopreservation on the developmental competence, ultrastructure and cytoskeletal structure of porcine oocytes. Molecular Reproduction and Development 73 1454-1462. 